\title{
Self-reproduction Cycles of Living Matter and Energetics of Human Activity
}

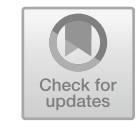

\author{
Leonid E. Popov
}

\begin{abstract}
In the author's opinion, many global problems that face humanity-in the fields of education, medicine, management etc. can be tackled more effectively if the cyclic nature of self-reproducing systems - including living beings-is taken into account. Summarizing the main physiological findings of the last decades on "adaptation reactions", one can very roughly say that the way of action which is effective in the sense of productive activity of people happens at the same time to be healthy, and it gives the participants of the process the feeling of happiness. The present paper represents a very short overview of the contemporary concepts of the adaptation reactions based on the fundamental understanding of their cyclic nature due to general properties of self-reproducing systems. One interesting feature of self-reproduction cycles is its first "phase of orientation" which was not discussed in detail in the past but plays a key role in the whole cycle.
\end{abstract}

Keywords Self-reproducing systems $\cdot$ Life $\cdot$ Adaptation reactions $\cdot$ Activation $\cdot$ Training $\cdot$ Stress $\cdot$ Health $\cdot$ Memory $\cdot$ Catabolic phase $\cdot$ Anabolic phase

\section{Introduction}

Any activity of living systems is cyclic at all levels of the organization of life. The cyclic character of activity of biological systems is associated not only with the periodic nature of the action of external factors on it (such as the rotation of the Earth around its axis, its movement around the Sun or the cyclic change in solar activity). The above mentioned cycles are the result of adaptation of living systems to periodic changes in the environment. However, life is cyclic in itself, by virtue of its very essence. Let us first briefly discuss this fundamental aspect of life which is essential for the following discussion.

How does "life" differ from inert matter? This question was put forward many times in the history of science. In retrospect, we can state that there is one point where

\section{E. Popov (殴}

Tomsk State University of Architecture and Construction, Pl. Solyanaya 2, 634003 Tomsk, Russia e-mail: 1.popov@vemn.de 
many researchers do agree: the essential property of living systems is their "reproduction". Let us consider this property closer. Chilean neuroscientists H. Maturana and F. Varela provide the following explanation: "When we speak of living beings, we presuppose something in common between them; otherwise we wouldn't put them in the same class we designate with the name 'living'.... Our proposition is that living beings are characterized in that, literally, they are continually self-producing. We indicate this process when we call the organization that defines them an autopoietic organization.

What is distinctive about...<living beings $>\ldots$ is that their organization is such that their only product is themselves, with no separation between producer and product. The being and doing of an autopoieic unity are inseparable, and this is their specific mode of organization" [1].

So living matter only exists due to continuously reproducing itself. The word "selfreproduction" very precisely reflects the meaning of the basic characteristic of all life: for all interactions with the environment, the living beings remain "themselves", although in the process of interaction parameters of a living system can significantly deviate from their values at steady state. The deviation is followed by a return to the stationary state of "stable non-equilibrium".

Self-reproduction means turning back to oneself. It would seem that there is no other interpretation here. But in the popular, as well as in scientific literature, selfreproduction is very often considered a synonym for reproduction of generations, and when speaking of "self-reproduction cycles", one usually means a cycle of producing a new generation. However, this aspect means the reproduction of population and the biological species as a whole, while each living unit reproduces itself continuously during its whole life. In the present paper, we focus of this-more unusual, but in reality central aspect of living things - their continuous self-reproduction during the course of individual life.

The process of individual self-reproduction is also cyclical in nature: after deviating from a stationary state, the system returns to the same state. Ugolev [2] formulated the principle of cyclic activity among the "principles of natural technologies of biological systems": "At all levels of organization (from cellular to planetary), biological systems (more precisely, processes in them) are partially or completely cyclized... The principle of recurrence is one of the most important principles to ensure maximum efficiency and effectiveness of living systems through the multiple use of the same structures. Cyclization also ensures coordination of all components, implementing a multi-stage process. Many process systems considered by us as linear will be later characterized as cyclic...".

\section{Three Phases of the Self-reproduction Cycle}

Thus, our life activity is discrete, and each cycle proceeds in phases. Let us first analyze the general structure of each such a cycle. Each behavioral act begins with the appearance in our mind of an image of the result of our action [3]. The formation 
of this image is an elementary event of prognostic activity of the nervous system. We can talk about the prognostic phase of the cycle. The two subsequent steps are obvious. The second phase is the action itself, leading to the achievement of the result using the functional system that was formed at the first stage. During this phase, the work is performed to achieve the desired (predicted) result, and this work is associated with the expenditure of free energy of the body. Finally, the third phase is the return of the organism to the steady state of stable disequilibrium. During this phase, the body recovers the energy and possibly structural costs related to the work done in the second phase.

We note two points here. Firstly, the formation of an image of the result of an organism's action is associated with the extraction of some information stored in long-term memory in the central nervous system. In addition, the formation of a functional system corresponding to this image requires preparation for the action of executive bodies. That is, the entire first phase of the cycle is associated with the processes of structure formation, with the processes of anabolism.

In the second phase, work is performed that is accompanied by the dissipation of free energy of the body, that is, with the cost of reserves of energy and, possibly, structure. Thus, the second phase is a catabolic one. The cost of energy and body structure is normally restored excessively in the third phase. Moreover, the processes of anabolism occur only in the structures that took part in the action.

Phenomenologically, the existence of the prognostic phase, during which the action is still absent, was of course known for a very long time, in various contexts, for example under the name of "keeping a clear head". "Keeping a clear head" in an acute situation does not mean not acting at all. "Clear head" refers first of all to the initial phase of orientation and forecast, without which the active action turns into "action nowhere." The absence of a phase of the formation of the image of the result of the action would lead to a chaotic, senseless and potentially dangerous activity for the body, the "panic". The physiological reaction characteristics of this first phase of the behavioral cycle have also been repeatedly described: The heart first "stops" (that is, the pulse drops sharply), and only then begins to "beat wildly". This physiological reaction can be found in such idiomatic expressions as a "heart-stopping event" (something that is very impressive or exciting).

However, it was this first phase of the cycle that was last to be discovered and described in the framework of physiological studies. A single cycle of physiological self-reproduction was studied by Arshavsky with collaborators in the laboratory of age-related physiology and pathology of animals and humans at the Institute of Experimental Medicine since 1935. By this time, researchers have already come to the understanding that living time is discrete (although it is perceived as flowing continuously): it is measured by metabolic cycles, which, as it was originally thought, consist of two phases: catabolic and anabolic. In the catabolic phase, motor activity is carried out, and work is done due to the energy of the destruction of cellular substrates. In the anabolic phase, the disturbed structure is restored, and recovery occurs to an extent exceeding the costs in the second phase. This transfers the body to a higher than the initial level of disequilibrium and ordering. 
Bearing in mind this excessive anabolism, I. A. Arshavsky called the metabolic cycle associated with a single motor activity a hypercycle.

But only in 1968 Arshavsky experimentally established the presence of another phase of the cycle: the "phase of the starting metabolism". Studies were conducted on the human fetus: through the wall of the abdomen of the mother, the fetal heartbeat was registered. It was expected that with recurrent "spontaneous" increase in activity would be associated increasing of the heart rate (catabolic processes occur with the dominance of the sympathetic nervous system that increases the heart rate), and then of slowing down (anabolism, dominance of the parasympathetic nervous system). It was found, however, that before each motor activity, the heartbeat slowed down (parasympathetic reaction, anabolism). After that, extensive research on puppies was undertaken during the process of prenatal development. On the obtained cardiograms, you can see all three phases associated with motor activity: a few rarer pulses (the starting anabolism phase), a rapid heartbeat during motor activity (catabolic phase) and a decrease in heart rate after the cessation of motor activity (phase of excessive anabolism).

In the fundamental work "Physiological mechanisms and regularities of individual development", where the results of research performed in the laboratory of age physiology and pathology in the 1930-1980s were summarized, I. A. Arshavsky gives the following definition of the adaptive response of the organism:

\begin{abstract}
Adaptation is the response of physiological-morphological transformation of the organism and its parts resulting in an increase of its structure-energy potential, that is, free energy and potential for action. This response is induced by such irritants of the environment which can be referred to as physiological, although they do demand the expenditure of an amount of energy. We call these irritants physiological because the energy expenditure resulting from them is compensated by its acquisition, that is by the functional induction of excess anabolism, automatically induced by each activity. As a consequence, a spiral-like transition of a developing organism to a higher level of potential working ability takes place. This adaptive response is characterized by three phases: the first phase is anabolic, the second one catabolic, giving the possibility of realizing another activity, and the third phase excessively anabolic. Speaking in terms of thermodynamics, we can interpret the above definition of adaptation as a response resulting in an increase of the free energy of the organism and ... the state of stable nonequilibrium... [4].
\end{abstract}

Note that the phase of excessive anabolism occurs only after activity. If there is no activity, then there is no development (and not even just recovery, since nonfunctioning cells self-eliminate by means of apoptosis mechanisms; an inactive organism degrades). It is in the phase of excessive anabolism that all development processes take place, in particular, the processes of long-term memory that stores our life experience. "...All motor activity, beginning with a zygote, is a factor of induction of anabolism, no matter if the motion is stimulated endogenously-due to the necessity of satisfying the demand for food, or exogenously-due to an irritant action of physiological stress. The purpose of this anabolism is not just recovery of the initial state, but necessarily excessive recovery. This transfers the system... to a new level, at which its internal energy increases... In the case of nervous cells it is manifested in trace hyperpolarization and ... in an increase in RNA content. For muscle cells it is trace hyperpolarization, trace hyperrelaxation, and an increase in 
protein content. It was found that ... anabolic excess is realized only due to activity. Experimental blocking of activity causes, in spite of continuing food intake, to a temporary stop or inhibition of growth and development, or even its stasis" [4].

Though I. A. Arshavsky, speaking of growth and development, means a growing organism, including the prenatal stage of its development, all of the above concerning the obligatoriness of function for excessive anabolism refers to any living creature independent of its age. In adult individuals, growth and development continue during their entire life in the form of expansion of the volume of long-term memory. The factors controlling the development of the nervous system of a growing organism and the processes within which this development is realized, are concentrated in the brain structures in adulthood, where new distributed multilevel systems of local neuron networks are formed (neocortex columns) in response to a sequence of signals about subjectively new realities of the environment. At present, the complete unity of the biochemical processes of development of the nervous system and of memory formation has been proven.

\section{Graphical Representation of Self-reproduction (Adaptation) Cycles}

Following [5], let us illustrate the above graphically. When we set ourselves the task of graphically representing the normal physiological adaptive response as a cyclic process, the value of structural energy of the organism $(F)$ is naturally assumed to be on the ordinate axis. It is suitable to quote here a comment made by I. A. Arshavsky concerning this quantity:

...the most essential qualitative feature of living systems is their state of nonequilibrium, steadily supported by the work of the structures comprising it-the work directed against their transition to the equilibrium state. The chemical energy of food substances entering the living systems can not directly transform into work. The energy, first and foremost, is used for producing free energy in the structure of the living system. In living systems, in contrast to nonliving ones, neither thermal nor chemical energy is the source of the work being performed by them, as is the case with thermo- and chemodynamical engines; the structural energy of the organism is responsible for that. The latter, in contrast to mechanical, thermal, chemical, electromagnetic, gravitational and nuclear energy is a specific form of energy inherent only in living systems [4].

Figure 1a illustrates the changes of the free structural energy during one typical cycle of self-reproduction. Point $A$ corresponds to the moment when an action on the organism requiring its response took place, point $B$ - the time of completion of the response, point $C$ - completion of the (excessively anabolic) phase 3 of the adaptive response. The dashed line shows the minimum value of free energy of the organism when it performs the work related to its adaptive response to the irritant. The figure illustrates the case when this limit is not exceeded: the free energy of the organism at point $B\left(F_{B}\right)$ is in excess of $F_{\min }\left(F_{B}>F_{\min }\right)$. In phase 3 , the organism returns 

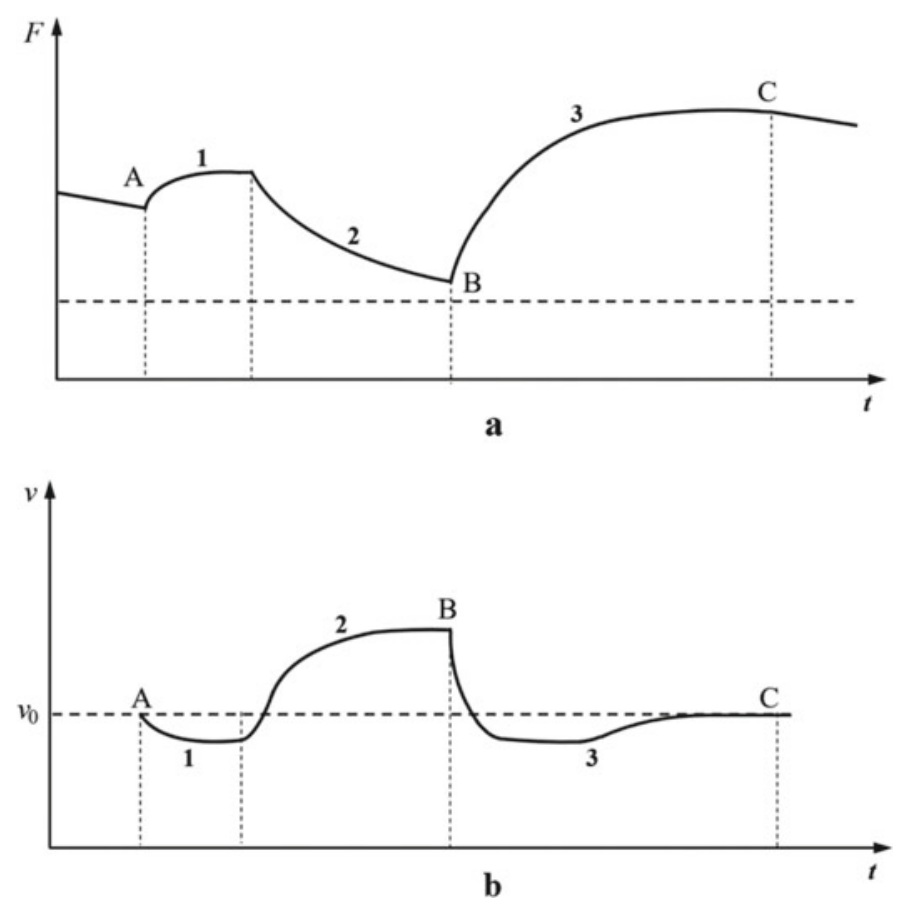

Fig. 1 a The change in the structural energy of an organism $(F)$ with time $(t)$ during an adaptive response (single cycle of self-reproduction). $\mathbf{b}$ The corresponding change in heart rate $(v)$ with time $(t)$

to a state of rest (point $C$ ), with higher free energy $F_{C}$ as compared with that of the initial state of rest.

Another important parameter of the cycle characterizing the deviation of the organism from the stable steady state is the heart rate $(v)$. One argument in favor of this choice is the circumstance that a change in the performance of the cardiovascular system in the conditions of catabolism and anabolism occurs in opposite ways. In the catabolic phase, the heart rate increases. In contrast, a decrease in the heart rate (vagal bradycardia [6]) is typical of anabolism. "... Vagal bradycardia is a necessary correlate both of the first and of the third anabolic phases of the physiological response of the entire organism. For the heart, the periodically occurring vagal bradycardia plays the role of providing the growth and development of the heart muscle. This is achieved through an increase in the duration of the diastolic pause ensuring an excess of regeneration processes upon every heart diastole" [4].

Facing a new stimulus, the organism first shows a temporary decrease of the heart rate (anabolic reaction associated with the prognostic phase) which is then followed by an increase of the heart rate in the phase of action, Fig. 1b. After achieving the result, the heart rate falls enabling excessive anabolism and restoration of the 
structural energy and finally comes to the equilibrium value-however, already with an increased level of structural energy.

Arshavsky's adaptive response results in the increase of structural energy of the organism:

$$
\Delta F=F_{C}-F_{A}>0
$$

and, consequently, an increase in its ability of performing work under following irritants of a similar (but not only) kind. I. A. Arshavsky referred to phase 3 of a normal physiological adaptive response as a phase of "excessive anabolism". This phase starts after performing a certain function at phases 1 and 2 by the organism and only in those structures which were involved in the realization of the function [4]. A dominating component of the adaptive response at these stages is motor activity both at the level of the whole organism (skeletal muscles), and at the cellular level [7].

\section{Hyper Cycles of Self-reproduction}

The cyclic nature becomes more visible if the free energy of the system is plotted as function of the heart rate, $v$.

In Fig. 2, Arshavsky's adaptive response is shown schematically in the coordinate system free energy $(F)$-heart rate $(v)$. Due to excessive character of anabolic stage, this is an open cycle ("hyper cycle" according to I. A. Arshavsky's terminology).

In Fig. 2, $v_{0}$ is the heart rate in the state of rest of a wakeful organism; point $A$ corresponds to the time of irritant action; point $B$ to the completion of necessary
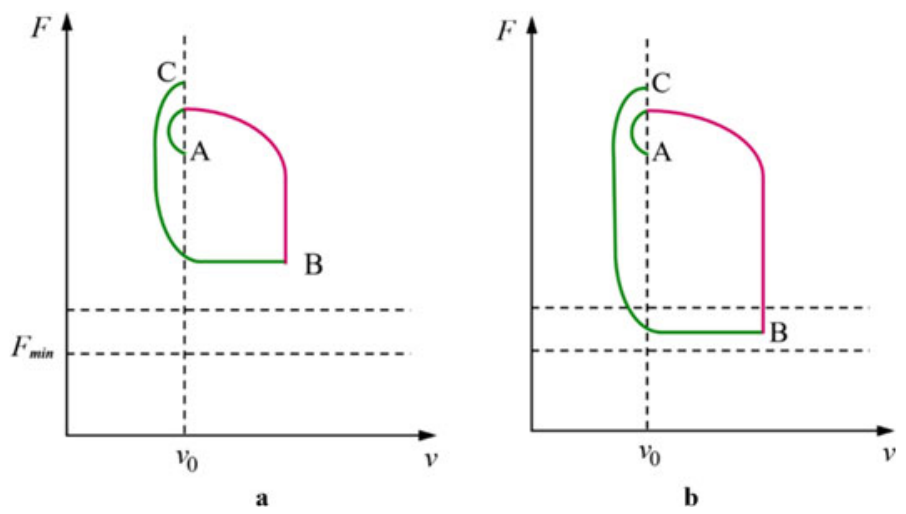

Fig. 2 Schematic of the adaptation response due to I. A. Arshavsky: $F$ - structural energy, $v$-heart rate. a Normal physiological response, $\mathbf{b}$ a critical physiological response 
responses; point $C$ to the completion of the third, anabolic, phase of the response and thereby the entire adaptive response.

Point $\mathrm{A}$ is the point at which we are caught up in the need to act. Immediately there is a decrease in heart rate and an increase in free energy in the prognostic phase, the formation of a functional system that will provide the subsequent action. Then this functional system acts (the region indicated by the red line and ending at point B). The action is followed by recovery, but the recovery is excessive, so that the body reaches a higher level of free energy than at the beginning. $F_{\min }$ is the energy, below which one should not go. Why? The reason is that during the "action" phase, carbohydrates are used as energy supply. If one continues to act, ignoring the capabilities of the body, the body will have to switch from carbohydrate to lipid metabolism, and then to protein metabolism. Horizontal dashed lines mark the area where lipid-protein metabolism is starting, (this is actually a certain transition region rather than a boundary). Thus, a healthy organism can sometimes use a part of its necessary structural resources (as shown in Fig. 2b) but restore this damage completely during the following excessive anabolic stage.

Depending on the strength of external influences, this cycle can be realized in several forms. For example, if the "problem to solve" turned out to be very simple and a solution was found with a minimum expenditure of resources, then at the completion of the action, the energy will not fall below the initial one (Fig. 3a). After this, however, the recovery phase still follows. All this happens within carbohydrate metabolism. Stronger impacts have already been discussed above.

And finally, if the irritant is too strong than the body is forced to enter the sphere of lipid-protein energetics (Fig. 3b). At the end of the action, in this case, the pulse drops, but not to the equilibrium value, and after that it very slowly returns to normal. Only after this the reverse transition to carbohydrate metabolism proceeds. After this, a
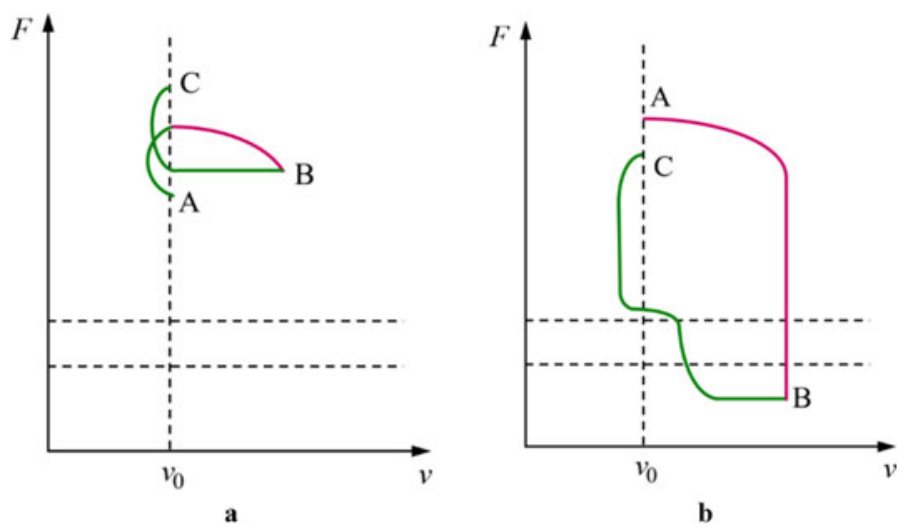

Fig. 3 Schematic of the adaptation response due to I. A. Arshavsky: $F$ - structural energy, $v$ - heart rate. a Response to weak stimuli (training reaction), b overcritical stimuli (pathogenic reactionstress) 
rapid decrease in pulse occurs, a transition to the stage of anabolism, which, however, may not be enough to completely restore the wasted structural energy.

\section{Non-specific Adaptive Responses}

The described cycles of self-reproduction are specific for each irritant. The particular nature of the irritant determines the image of the result of the action as well as the structures of the organism comprising the functional system formed in the phase 1 and the duration of the activity needed to achieve the result. However, not always the result can be achieved in one cycle so that several cycles may be required. In conditions of prolonged focused activity, a general non-specific adaptive response of the body is formed, controlled by the hypothalamus-hypophysis-adrenal gland system $[4,8,9]$. Depending on the strength of the stimulus, it may be a "training reaction" [8], an "activation reaction" [8] or an enhanced activation reaction [10]. Finally, in a situation where efforts to achieve the result are required that exceed the physiological capabilities of the body, a pathological response develops-stress $[8,11]$.

Apparently, one of the listed adaptation answers corresponds to the dominance of the long-term purposeful activity of each of the options for the elementary reproduction cycle described above.

The four non-specific adaptation reactions are summarized in Fig. 4, which shows the power of vital activity (consumption of structural energy per unit time) versus time.

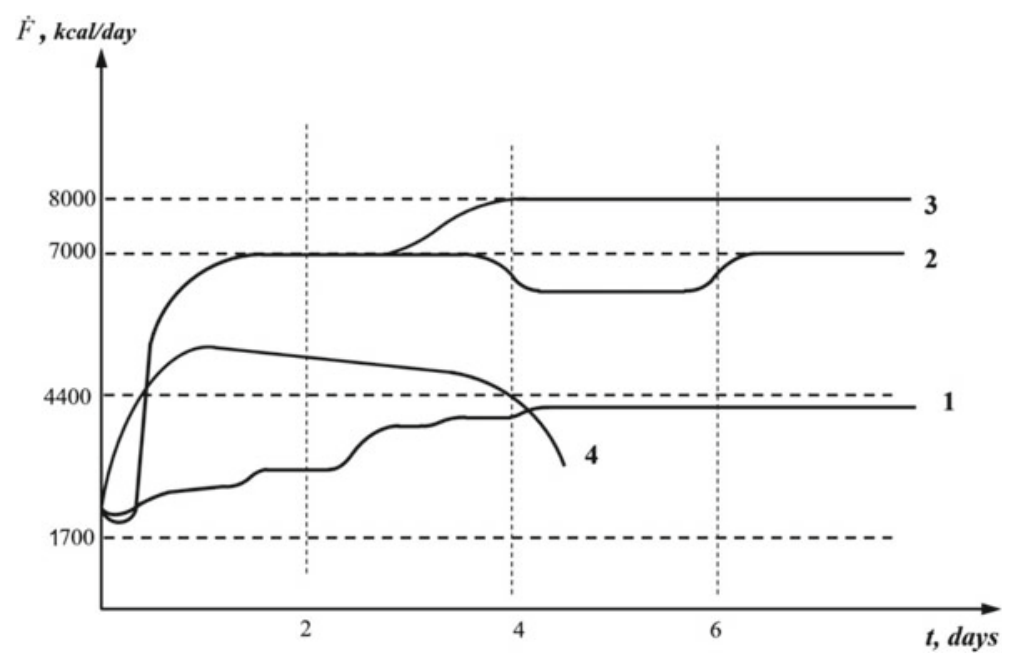

Fig. 4 Metabolic power characteristic for: 1: weak stimuli (training reaction), 2: average stimuli (activation reaction), 3: enhanced activation, 4: stress 
Curve 1 corresponds to the case of weak stimuli (Fig. 3a). The maximum metabolic power developed by humans in the state of this "training response" to weak stimuli does not exceed $4400 \mathrm{kcal}$ per day [12].

The second option corresponds to the so-called activation reaction [12]. Being in this state is very becoming to the organism. Changes in the immune system accompanying this response occur in the direction of increasing the organism's resistance, improving the immune systems. Without going into detail about the mechanisms of formation of the activation response ("physiological stress" according to I. A. Arshavsky), we should note that the adaptive responses of the organism are largely controlled by hormonal activity of the hypothalamus-hypophysis-adrenalgland system. When the organism functions in the regime of the Arshavsky's adaptive response (activation response according to Ukolova [8]), the system releases anabolic agents into the bloodstream: growth-hormone-releasing factor of the hypothalamus, somatotropic hormone of the hypophysis, mineralcorticoids and testosterone of the adrenal gland, etc. In general, the processes of anabolism, recovery, and development dominate in the organism in this case. This is facilitated by the fact that the activation reaction is accompanied by a certain decrease in blood coagulability, which ensures high mobility of changes in blood supply (and therefore nutrition) of all functional tissues of the organism.

During the activation response a human can use up to $7000 \mathrm{kcal}$ per day [12]. For comparison, recall that the base metabolism rate required for sustaining the vital activity of the organism in a state of complete rest is approximately $1600 \mathrm{kcal}$ per day, and in our ordinary vital activity, including laborious activity, we use about $3500 \mathrm{kcal}$ per day.

In the case of prolonged activity, long and intense work is possible within the framework of a reaction of enhanced activation with a power reaching $8000 \mathrm{kcal}$ per day.

\section{Stress and Necessity Avoiding It}

However, in reality, man often finds himself in situations requiring efforts that exceed the physiological capabilities of the organism. A response to extreme irritants ("strong stimuli" $[8,10]$ ) allows the organism to survive as a living system, but leaves behind injuries and pathological changes. This modus of activity is shown in Fig. 4 as line 4 .

The anabolic stage under stress is weak or is entirely absent [4] (Fig. 3b), while the catabolic stage 2 leads to expenditures of structural energy and to structural changes in the organism that exceed its regenerative abilities, the increase in structure energy at stage 3 is lower than the expenditures at the catabolic stage 2: $\Delta F=F_{C}-F_{A}<$ 0 . This adaptive response of animal and human organisms to excessive irritants was discovered earlier than the responses corresponding to normal physiology by Canadian physiologist H. Selye in 1936 [9]. He called the response stress (the term became widely used mostly owing to journalists who used and abused the word). The changes 
in the hypothalamus-hypophysis-adrenal-gland system controlling the hormonal status of the organism, as Selye $[9,11]$ and other researchers have shown, are of a mobilizatory character. The hypothalamus, secreting the corticotropic-releasing factor into the blood vessels of the hypophysis triggers the release of adrenocorticotropic (ACTH) hormone by the hypophysis, which in turn induces secretion of corticosteroids by the adrenal cortex, along with the release of catecholamines, adrenaline and noradrenaline, by the adrenal medulla. Both corticosteroids and catecholamines are factors whose function is the emergency mobilization of the organism. Energy is mostly generated on the lipid (adipose) basis. Carbohydrate energy is suppressed. Physiologists even have a proverb: "Fats burn in the carbohydrate fire, but carbohydrates do not burn in the fat fire" [8]. This is a very important circumstance, since the nervous system functions only on the basis of carbohydrate energy. Therefore, while in stress, the cognitive component of vital activity is either suppressed or absent. In other words, stress actions are unacceptable in the educational space and in educational cognitive activity. When designing educational technologies, the pathological adaptive response, stress, should be considered only insofar that it must be eliminated.

Let us note some other features of the pathological adaptive response. An increase in coagulability occurs (a natural evolutionary adaptation: stress situations may result in bloodshed). Blood circulation becomes less dynamic, tissue nutrition is decreased or stopped, including that of functional tissues. One of the characteristic stress symptoms is the formation of multiple bleeding ulcers in the digestive system [8]. Thus, stress never does without bleeding.

The immune system is literally switched off. Its major organ, the thymus, drastically decreases in size, all immune factors are released into the bloodstream, and as a result the organism is relatively resistant to pathogenic influences for three days. But in the stage of exhaustion which sets in on the third or fourth day of stress, the human becomes ill. And although the "weak links" of the organism differ from person to person, in most cases acute tonsillitis occurs, due to the defenselessness of the tonsils, which are lymph-glands, and therefore, a part of the immune system. In any case, if one finds that the mandibular glands are swollen, one should try to remember what trouble happened 3 days ago, and by revealing the stressor, eliminate it, changing the attitude towards it or by restructuring near-term plans.

It should be noted that under stress the water-mineral metabolism changes so much that the weight of a human increases by $2 \mathrm{~kg}$ in several hours [12]. Therefore, a person who regularly observes their weight can easily discover stress by weighing.

\section{Conclusions}

We provided a brief review of adaptation reactions stressing the importance of their cyclic nature as well as the first anabolic phase of a cycle (prognostic phase, when the image of the result of action and the action itself is created). The central role in the self-reproducing cycles is played by the anabolic phases. Normally, one of the 
anabolic phases follows a catabolic phase during which the resources of the organism are expended. However, in exceptional cases, it is possible that the whole activity remains anabolic.

We discuss the importance of avoiding stress reaction-both in terms of health and educational activities (as stress never leads to development of long-term memory).

In the past years, the author discussed some of this topics with S. G. Psakhie in context of creative scientific activity and its organization.

Acknowledgements The author is thankful to V. L. Popov for discussions of the key ideas of the present paper and for his assistance in preparing the manuscript, Elena Komar' for preparing illustrations and M. Popov for proofreading the Chapter.

\section{References}

1. Marturana HR, Varela FJ (1987) The tree of knowledge: the biological roots of human understanding. New Science Library/Shambhala Publications, Boston, $263 \mathrm{pp}$

2. Ugolev AM (1987) Natural technologies of biological systems. Nauka, Leningrad, 318 p (in Russian: 317 c.)

3. Anokhin PK (1968) Biology and neurophysiology of conditional reflex. Medicine, Moscow, p 546 (in Russian)

4. Arshavskii IA (1982) Physiological mechanisms and regularities of individual development. Nauka, Moscow, p 270

5. Popov LE, Postnikov SN, Kolupaeva SN, Slobodskoy MI (2015) Natural resources and technologies in educational activities: education in times of accelerated technological development. Cambridge International Science Publishing Ltd., Cambridge, 133 pp

6. Semenova ST (1993) Vernadsky and Russian Cosmism, Vladimir Vernadsky, Biography. Selecta. Memory Lane of Contemporaries. Descendant Speculations. Sovremennik, Moscow, pp 596-646

7. Penrose R (1994) Shadows of the mind: a search for the missing science of consciousness. Oxford University Press, Oxford, p 457

8. Garkavi LH, Kvakina EB, Ukolova MA (1977) Adaptive responses and resistance of the organism. Rostov University Press, Rostov-on-Don, p 126

9. Selye $\mathrm{H}$ (1936) Thymus and adrenals in the response of the organism in injuries and intoxication. Br J Exp Pathol 17(3):234-248

10. Garkavi LH, Kvakina EB, Kuz'menko TS (1998) Antistress responses and activation therapy. IMEDIS, Moscow, p 656

11. Selye H (1972) At the level of the whole organism. Nauka, Moscow, p 122

12. Hammond KA, Diamond JM (1997) Maximum sustained energy budgets in humans and animals. Nature 386:457-462 
Open Access This chapter is licensed under the terms of the Creative Commons Attribution 4.0 International License (http://creativecommons.org/licenses/by/4.0/), which permits use, sharing, adaptation, distribution and reproduction in any medium or format, as long as you give appropriate credit to the original author(s) and the source, provide a link to the Creative Commons license and indicate if changes were made.

The images or other third party material in this chapter are included in the chapter's Creative Commons license, unless indicated otherwise in a credit line to the material. If material is not included in the chapter's Creative Commons license and your intended use is not permitted by statutory regulation or exceeds the permitted use, you will need to obtain permission directly from the copyright holder.

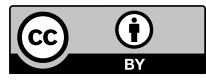

